\title{
Why cholesterol ester transfer protein (CETP) inhibitors have and will likely continue to fail in trials undertaken to increase blood concentrations of HDL-cholesterol and reduce cardiovascular events
}

\begin{abstract}
Sander J. Robins*1
Epidemiologic studies have consistently shown that increased blood concentrations of high density lipoprotein-cholesterol (HDL-C) are associated, independent of other well-established cardiovascular disease (CVD) risk factors, with a reduced incidence of major CVD events. Conversely, low concentrations of HDL-C have been long recognized as a major and independent CVD risk factor.
\end{abstract}

${ }^{1}$ Professor of Medicine, Boston University School of Medicine, Laboratory Director, Framingham Heart Study, 73 Mt Wayte Ave, Framingham, MA 01702, USA

Over the past decade there has been a considerable effort to pharmacologically increase HDL-C concentrations, most recently on a relatively large scale with the administration of cholesteryl ester transfer protein (CETP) inhibitors which, although substantially increasing blood levels of HDL-C in what to-date have been six large clinical trials, with a cumulative trial population of about 75,000 subjects, have, in none of these trials, as identified below, succeeded in reducing major CVD events.

As a result of these trial failures there have been commentaries such as one titled "Is raising HDL a futile strategy for atheroprotection?" $[1,2]$ which concluded "the dramatic failure of clinical trials evaluating the cholesterol ester transfer protein inhibitor torcetrapib has led to considerable doubt about the value of raising high-density lipoprotein cholesterol (HDL-C) as a treatment for cardiovascular disease".

This and other like attempts to explain the failings of CETP inhibition trials appear not to give emphasis to the difference in cholesterol $(\mathrm{CH})$ that is present in the circulation in association with HDL as an unesterified molecule with "sparing water-solubility" (as possibly a microcrystalline dispersion) compared to a larger molecule where $\mathrm{CH}$ is esterified with a long-chain fatty acid with virtually no solubility in water and is more distinctly a "lipid" molecule.

The major route of $\mathrm{CH}$ elimination from the body is by hepatobiliary secretion, a process that in an animal study has been shown to, most expeditiously, take place along a direct intra-hepatic canalicular membrane route [3]. It is well established that only unesterified (or "free") sterols have the physical properties to be solubilized within a bile acid micelle to be secreted in bile [4]. Indeed, there appears to be no known excretory pathway for $\mathrm{CH}$ esters from the liver nor is there any specific information regarding the fate of esterified $\mathrm{CH}$ taken up by the hepatic SR-BI receptor, most particularly, with respect to its possible conversion within the liver back to "free" $\mathrm{CH}$ to then find its way through the liver to be "channeled" into bile.

Given the specific solubility requirements for the secretion of $\mathrm{CH}$ in bile it might be anticipated that increasing blood concentrations of HDL-CE (with CETP inhibitor therapies) would, therefore, not provide a $\mathrm{CH}$ substrate that might be "solubilized" in a bile acid/ phospholipid micelle to be secreted from the body in bile. Indeed, this appears to have been now largely proven to be the case by six published human trials (titled ILLUMINATE, RADIANCE, REVEAL, DEFINE, ACCELERATE, and dal-OUTCOMES), undertaken with one of four different CETP inhibitor drugs, that have each substantially increased blood concentrations of HDL-CH as, specifically, HDLCE (for example, by $40 \%$ with dalceptrapib to as much as $130 \%$ with evacetrapib), but that have, without seemingly any certain explanation, uniformly failed to reduce major CVD events [5,6].

A number of years ago it was not unusual for clinical laboratories to more regularly measure plasma concentrations of both unesterified $\mathrm{CH}$ and total $\mathrm{CH}$, from which one could then readily calculate an amount of CE. What the failure of CETP inhibitor therapy may also be suggesting is that it may possibly have been a "mistake" for laboratories to have stopped (often routinely) measuring blood levels of both HDLunesterified (HDL-UC) and esterified (HDL-CE) cholesterol since these two different lipoprotein particles do not just have a different chemical and physical structure, but have very different biologic properties that may well have different implications for cardiovascular outcomes.

A possible revision of $\mathrm{CH}$-directed therapy that would seem to be in accord with what we know to be the positive properties of unesterified $\mathrm{CH}$, most particularly, the necessity of UC as a stabilizing component of all phospholipid-structured animal cell membranes, would seem to be, if anything, to at this time inhibit the action of CETP with the aim of increasing plasma concentrations of HDL-UC.

Perhaps of additional importance is that this large amount of past (and "negative") CETP trial data would appear to further suggest that our characterization of $\mathrm{CH}$ as a CVD "risk factor" needs to be now more explicitly defined to include when HDL-C is a clinical focus, the measurement of, most particularly, blood concentrations of HDL-UC, that may more directly relate to the medical consequences of a major

Correspondence to: Sander J. Robins, Professor of Medicine, Boston University School of Medicine, Laboratory Director, Framingham Heart Study, $73 \mathrm{Mt}$ Wayte Ave, Framingham, MA 01702, USA, Tel: (508) 935-3452, Fax: (508) 8200340, E-mail: sjrobins@bu.edu

Received: March 16, 2017; Accepted: April 07, 2017; Published: April 10, 2017 
CVD event then the totality of HDL-C concentrations that includes cholesteryl esters. For the public it seems sufficient to indicate that there are two kinds of HDL-C with ONLY one kind that has been shown to be truly cardio-protective which is "HDL-UC" and this, ideally, might well be independently measured and reported, apart from the "total HDL-CH" which should be less predictive in assessing CVD risk.

It might be noted that the word "cholesterol" is derived from the ancient Greek "khole" (or "chole") steros" which was used to give name to the initial finding of a $\mathrm{CH}$ gallstone within the biliary tract. Coincidently, it is clearly a hepato-biliary route that is the only certain route for $\mathrm{CH}$ elimination from the body in humans, and it is one that most particularly requires a $\mathrm{CH}$ molecule that is unesterified in order to be incorporated within a bile acid micelle that is requisite for its secretion in bile.

\section{References}

1. Joy T, Hegele RA (2008) Is raising HDL a futile strategy for atheroprotection? Nat Rev Drug Discov 7: 143-55. [Crossref]

2. Robins SJ, Fasulo JM (1997) High density lipoproteins, but not other lipoproteins, provide a vehicle for sterol transport to bile. J Clin Invest 99: 380-384. [Crossref]

3. Robins SJ, JM Fasulo (1999) Delineation of a novel hepatic route for the selective transfer of unesterified sterols from HDL to bile. Studies using the perfused rat liver. Hepatology 29: 1541-1548. [Crossref]

4. Small DM (1986) The Physical Chemistry of Lipids. pg 406, Plenum Press, NY and London.

5. Robins SJ, Lyass A, Brocia R, Massaro JM, Vasan RS (2013) Plasma lipid transfe proteins and the development of cardiovascular disease. The Framingham Heart Study. Arteriosclerosis 228: 230-236. [Crossref]

6. Robins SJ, Fasulo JM, Prizker CR, Patton GM (1996) Hepatic transport and secretion of unesterified cholesterol in the rat is traced by the plant sterol, sitostanol. J Lipid Res 37: 15-21. [Crossref]

Copyright: (C2017 Robins SJ. This is an open-access article distributed under the terms of the Creative Commons Attribution License, which permits unrestricted use, distribution, and reproduction in any medium, provided the original author and source are credited. 